\title{
O ACESSO À ATENÇÃO PRIMÁRIA À SAÚDE: FATORES FACILITADORES
}

Nicássia Moro Roce, Márcia Farsura de Oliveira, Lorena Souza e Silva. O acesso à atenção primária à saúde: fatores e facilitadores. Revista Saúde Dinâmica, vol. 2, núm.3, 2020. Faculdade Dinâmica do Vale do Piranga.

\section{SAÚDE DINÂMICA - Revista Científica Eletrônica FACULDADE DINÂMICA DO VALE DO PIRANGA}

5a Edição 2020 | Ano II - no 3 | ISSN-2675-133X 


\title{
O acesso à atenção primária à saúde: fatores facilitadores
}

\section{The access to primary health care: facilitating factors}

\author{
Nicássia Moro Roce ${ }^{1}$, Márcia Farsura de Oliveira ${ }^{2}$, Lorena Souza e Silva ${ }^{3}$ \\ ${ }^{1}$ Discente do Curso de Medicina, Faculdade Dinâmica do Vale do Piranga, https://orcid.org/0000-0002-7883-7391 \\ ${ }^{2}$ Docente no Curso de Medicina, Faculdade Dinâmica do Vale do Piranga, https://orcid.org/0000-0001-8462-0431 \\ ${ }^{3}$ Docente no Curso de Medicina, Faculdade Dinâmica do Vale do Piranga, https://orcid.org/0000-0001-7355-7510 \\ Autor correspondente: nicassiamr@gmail.com
}

\section{Resumo}

A Constituição da República Federativa do Brasil reconhece o termo saúde como uma concepção ampla, um direito universal de cidadania, devendo oferecer condições do acesso universal através de políticas públicas, as quais recomendam que o paciente adentre os serviços de saúde pela assistência primária, onde é resolvida a maioria dos problemas, e seja encaminhado, quando necessário, aos serviços de maior complexidade. O presente artigo objetiva identificar os fatores facilitadores do acesso à Atenção Primária à Saúde (APS), visando propor melhorias no atendimento, a partir de uma revisão integrativa de literatura com um levantamento bibliográfico nas bases de dados LILACS e Scielo, utilizando os descritores "Atenção primária à saúde" e "Acesso aos serviços de saúde". Os resultados identificados na análise crítica que abordavam o tema em questão, foram: o acolhimento; a implantação da Estratégia Saúde da Família (ESF); a efetivação de Redes de Atenção à Saúde; e o gerenciamento da Unidade Básica de Saúde (UBS). Ademais, observou-se nesse estudo, que as as ações de saúde intencionam alcançar a população plenamente, investindo no acesso do paciente aos serviços de saúde. No entanto, pesquisas em condutas que contribuam para a saúde pública são escassas, assim como as dificuldades vivenciadas pelos recursos humanos, para a ruptura de paradigmas presentes na prática.

Palavras-chave: Atenção primária à saúde; Acesso aos serviços de saúde; Integralidade em saúde.

\begin{abstract}
The Constitution of the Federative Republic of Brazil recognizes the term health as a broad concept, as well as a universal right of citizenship, offering conditions that support universal access through public policies, which recommend that the patient enters health services through primary care, where most problems are solved and later forwarded to services of greater complexity, when necessary. The present article's objective is to identify the factors that facilitate access to Primary Health Care (PHC), aiming to propose improvements in citizen care, based on an integrative literature revision with a bibliographic search carried out in the LILACS and Scielo databases, utilizing the descriptors "Primary health care" and "Access to health services". The results identified throughout the critical analysis of the topic in question were: the reception; the implementation of the Family Health Strategy (FHS); the establishment of a Health Care Networks; and the management of the Basic Health Unit (BHU). Furthermore, throughout the study it was observed that the health actions intend to reach the population to its full extent, investing in the patient's access to health services. However, research on conducts that contribute to public health are scarce, as well as the difficulties experienced by human resources, for the rupture of paradigms present in health practices

Key words: Primary health care; Access to health services; Integrality in health.
\end{abstract}




\section{INTRODUÇÃO}

A primeira Conferência Internacional sobre Cuidados Primários de Saúde foi realizada em setembro de 1978, na República do Cazaquistão, resultando na Declaração de Alma-Ata, que reforçou o significado da saúde como um direito fundamental do ser humano (MENDES, 2004; STARFIELD, 2002).

Nesse cenário, antes da criação do Sistema Único de Saúde, a assistência à saúde era ofertada aos que podiam pagar pelo serviço ou aos trabalhadores da economia formal, ou seja, não era universal. Aqueles que não se enquadravam nessas categorias viviam a mercê de instituições filantrópicas e caridade (DE SOUZA, 2002).

Em 1988, a Constituição da República Federativa do Brasil reconhece o termo saúde como uma concepção ampla, como um direito universal de cidadania, resultante de condições de vida e trabalho e inserida no plano das políticas sociais. Segundo a Constituição Federal, a saúde é:

"Direito fundamental do ser humano, devendo o Estado prover as condições indispensáveis ao seu pleno exercício através de políticas sociais e econômicas e do estabelecimento de condições que assegurem acesso universal e igualitário às ações e aos serviços para sua promoção, proteção e recuperação" (BRASIL, 1988).

A partir daí, com a promulgação da Constituição da República Federativa do Brasil, é instituído na seção II, o tópico Saúde, versando sobre o Sistema Único de Saúde (SUS), que passa a ser regulamentado pelas Leis número 8.080 e 8.142, ambas de 1990 (BRASIL, 1988; SILVA; RASERA, 2014).

A Lei 8.080 de 19 de setembro de 1990, “dispõe sobre as condições para promoção, proteção e recuperação da saúde"; enquanto a Lei 8.142 de 28 de dezembro de 1990, estabelece a participação da comunidade na gestão do SUS (ASSOCIAÇÃO PAULISTA DE MEDICINA, 2008; SILVA; RASERA, 2014). Essas regulamentações passam, a partir de suas promulgações, a regular as ações e serviços de saúde e organizam a participação popular na gestão e fiscalização desses serviços (ASSOCIAÇÃO PAULISTA DE MEDICINA, 2008).

Com isso, a saúde passou a ser uma condição garantida a todos os cidadãos pelo Estado, dentro de um sistema fundamentado por princípios (i) doutrinários: universalidade, equidade e 
integralidade; e (ii) organizativos: descentralização, participação social, regionalização e hierarquização do sistema; os quais apontam para a democratização das ações e serviços de saúde (AGUIAR, 2015; DE MATTOS, 2009).

De acordo com a Lei 8.080, a universalidade, princípio doutrinário do SUS que define que os serviços públicos de saúde sejam de livre acesso a todos os cidadãos, estabelece uma garantia de assistência e acesso à saúde para a população em todos os níveis de atenção (BRASIL, 1990; AGUIAR, 2015). O princípio da equidade refere-se em tratar desigualmente os desiguais, buscando, de certa forma, corrigir as mazelas sociais e em saúde (AGUIAR, 2015; STARFIELD, 2002). Já a integralidade, considera o ser humano com um todo, vinculando a promoção, prevenção, tratamento e reabilitação no cuidado prestado, abrangendo assim todas as suas necessidades biopsicossociais (AGUIAR, 2015; STARFIELD, 2002).

Dentre os princípios organizativos do SUS, a descentralização estabelece a divisão da responsabilidade entre os níveis municipal, estadual e federal. A participação social é uma garantia da sociedade para que a população atue no processo de construção, controle e execução de políticas públicas. A regionalização e hierarquização, estão relacionadas com os diferentes níveis de atenção - primário, secundário ou terciário - e a relação estabelecida entre esses serviços (AGUIAR, 2015; DE MATTOS, 2009; STARFIELD, 2002).

A descentralização tem como prioridade a corresponsabilidade nas ações e serviços de saúde nos três níveis governamentais, com ênfase na municipalização. Com isso, o município passa a ser o principal responsável pela saúde populacional, em que os gestores, ao receberem os recursos financeiros, possuem autonomia para "coordenação, negociação, planejamento, acompanhamento, avaliação e controle sobre os recursos, assim como, sobre as ações e serviços de saúde do seu território" (AGUIAR, 2015, p. 73).

Essa forma de gerir é importante, pois cada município apresenta uma realidade, sendo desenvolvidas diferentes políticas de saúde específicas para cada situação vigente. Ademais, a descentralização está diretamente relacionada a uma organização regionalizada e hierarquizada, para uma melhor integração das redes de serviços. Assim, inicialmente, o paciente adentra os serviços de saúde através da assistência primária, onde são resolvidos a maior parte dos problemas, e posteriormente encaminhados, quando necessário, aos serviços de maior complexidade tecnológica (AGUIAR, 2015; CORDOBA, 2013).

Entretanto, com relação aos níveis de assistência, a atenção primária à saúde (APS) tem papel fundamental no acompanhamento do paciente, pois trata-se do ingresso para o cidadão 
dispor das atividades em saúde e primeiro contato do paciente com o SUS. Desempenha funções, como buscar melhoria na qualidade de vida da população, através de ações de prevenção, promoção, tratamento e reabilitação, além do acesso universal e contínuo aos serviços de saúde de forma interdisciplinar, visando o acompanhamento integral do paciente. Para mais, aborda problemas comuns na sociedade a fim de ampliar a saúde e o bem-estar, por meio de ações focadas na prevenção e promoção de saúde. (AGUIAR, 2015; STARFIELD, 2002).

A atenção secundária é utilizada em casos de média complexidade, principalmente por ambulatórios de especialidades e hospitais de pequeno e médio porte. Enquanto a atenção terciária, é ofertada por hospitais gerais especializados de grande complexidade e alta tecnologia (CORDOBA, 2013).

Com o intuito de melhorar o sistema de serviços de saúde foram desenvolvidas as Redes de Atenção à Saúde, prestando uma assistência contínua e integral a população, para interligar os três níveis de saúde quando necessário (BRASIL, 2010b).

As redes de atenção possuem uma forma de organização poliárquica, na qual a coordenação é realizada pela APS, com enfoque nas condições agudas e crônicas através de uma rede integrada de pontos de cuidado à saúde. Apresenta como objetivo a melhoria da saúde na comunidade, dividindo a responsabilidade entre os níveis de atenção e o indivíduo (BRASIL, 2010b).

Nesse contexto, para a efetivação dos princípios do SUS em todos os níveis de atenção em saúde, foi criada a Política Nacional de Humanização (PNH), desenvolvida em 2003. Tal política foi elaborada a fim de melhorar a comunicação entre gestores, trabalhadores e usuários da saúde pública, incentivando o aprimoramento do cuidado, da atenção e produção de saúde (BARBOSA et al., 2013). Dessa forma, o ensino teórico e prático passa a ser aliado no desenvolvimento da educação continuada, atualização de procedimentos técnicos, avaliação e evolução de quadros clínicos, visando a renovação e inovação constantes no atendimento do paciente pelos profissionais de saúde (BARBOSA et al., 2013).

O diálogo entre os atores envolvidos pode proporcionar soluções para os diversos obstáculos que os usuários dos serviços de saúde apontam como complicadores do acesso, tais como, os horários de atendimento e a morosidade na marcação das consultas (LIMA, 2015). Por outro lado, também surgem questões sobre a importância da construção do vínculo entre a 
equipe de saúde e o paciente, com o propósito de aproximar e facilitar o acesso à APS (SILVA et al., 2018).

Dessa forma, devido ao fato de muitos pacientes não se sentirem acolhidos ou não estabelecerem um vínculo na unidade básica de saúde da família da qual fazem parte, torna-se importante o entendimento dos fatores que facilitam o primeiro contato dos usuários com a APS. Neste contexto, o objetivo central da pesquisa foi realizar uma revisão sistemática sobre os fatores facilitadores do acesso à Atenção Primária, a fim de identificar e divulgar melhorias no atendimento aos cidadãos e fazer com que estes sintam segurança e confiança ao buscarem assistência na APS.

\section{MATERIAIS E MÉTODOS}

O presente trabalho consiste em uma revisão integrativa de literatura, por meio de levantamento bibliográfico de artigos nacionais publicados nos últimos dezesseis anos que tratam sobre os temas: Atenção primária à saúde e Acesso aos serviços de saúde. A escolha do período de dezesseis anos se justifica pela criação e implementação da Política Nacional de Humanização em 2003, sendo interessante realizar a busca de dados a partir deste momento. Este levantamento ocorreu durante os meses de setembro de 2019 e janeiro de 2020 e a revisão integrativa foi realizada a partir de seis etapas.

A primeira etapa abarcou a definição do tema, dos objetivos do estudo, definição das palavras-chaves e seleção da questão norteadora da pesquisa: “O acesso à Atenção Primária à saúde: fatores facilitadores".

Na segunda etapa, um levantamento bibliográfico foi realizado nas bases de dados LILACS (Literatura Latino Americana e do Caribe em Ciências Sociais e da Saúde) e Scielo (Scientific Eletronic Library OnLine). A busca teve como critérios de inclusão artigos originais e de revisão publicados entre os anos de 2003 a 2019, na língua portuguesa e disponíveis online sob a forma de texto completo, utilizando os descritores "Atenção primária à saúde" e "Acesso aos serviços de saúde" como assuntos principais. Por meio da aplicação destes filtros de busca 233 artigos foram encontrados, onde 139 deles foram obtidos na base LILACS e 94 na base Scielo. 
Na terceira etapa, foi efetuada a análise seletiva dos artigos, através da leitura dos títulos, resumos, e palavras-chave dos artigos selecionados na etapa anterior, tendo sido excluídos nesta fase os artigos que não abordaram o propósito da revisão integrativa, bem como os que se encontraram em duplicidade, totalizando na seleção de 28 artigos, sendo 15 selecionados na base de dados LILAC e 13 na base de dados Scielo.

$\mathrm{Na}$ quarta etapa, momento de análise crítica, os 28 artigos pré-selecionados foram analisados por leitura dos textos na íntegra, buscando delimitar as categorias de análise, e responder aos objetivos da pesquisa, observando que 14 trabalhos respondiam à questão norteadora da revisão. Destes, 6 foram selecionados na base LILACS e 8 na base Scielo.

Na quinta etapa, foi realizada a interpretação qualitativa dos dados, e por fim, na sexta e última etapa, realizou-se análise crítica dos dados, abrangendo a discussão das informações e evidências encontradas.

Além dos artigos selecionados conforme as etapas descritas, também fizeram parte da revisão integrativa, a Constituição da República Federativa do Brasil de 1988, livros, decretos, leis e informações relacionados à questão norteadora da pesquisa, disponíveis em sites especializados, como o sítio eletrônico do Ministério da Saúde. 
Figura 1 - Fluxograma do processo de seleção dos estudos para a composição da revisão integrativa.

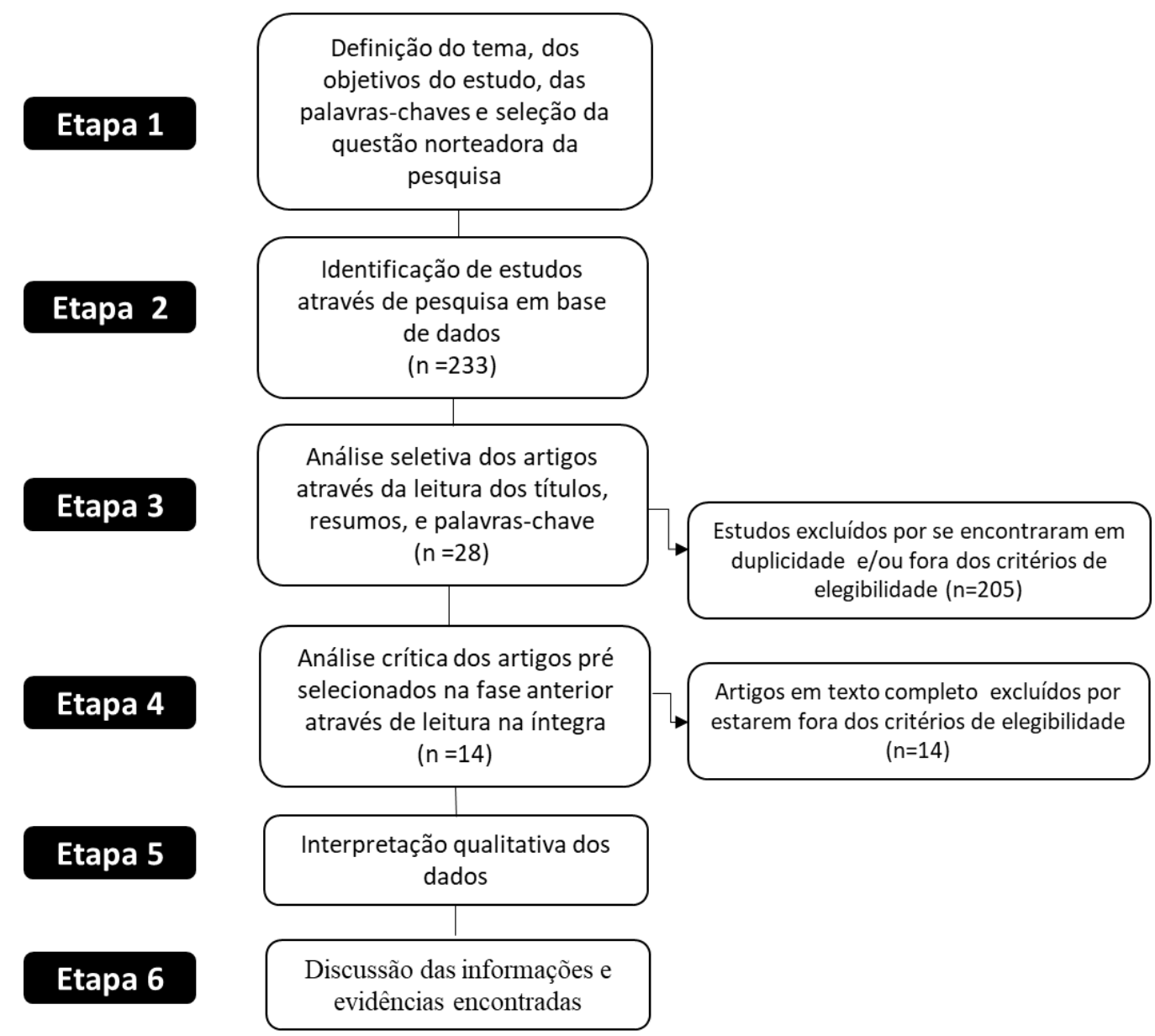

Fonte: Elaboração própria.

\section{RESULTADOS E DISCUSSÃO}

Quanto às características gerais, a publicação mais antiga encontrada foi de 2008 e a mais recente de 2019, a busca através do descritor "Atenção Primária à saúde" resultou em 5 artigos elegíveis para a construção da presente revisão e a busca através do descritor “Acesso aos serviços de saúde" resultou em 9 artigos.

O grupo amostral selecionado nos artigos incluídos nesta revisão, englobaram deste os usuários do sistema de saúde de uma forma geral, bem como populações específicas como 
cuidadores de crianças e a população masculina; a principal ferramenta metodológica empregada nos artigos selecionados foram as revisões sistemáticas e integrativas, com exceção de um único estudo, que adotou a entrevista como metodologia e o Instrumento de Avaliação da Atenção Primária, o Primary Care Assessment Tool (PCATool) como ferramenta de coleta de dados.

Todos os trabalhos escolhidos para a construção da revisão integrativa, encontram-se sintetizados no Quadro 1 abaixo, o qual traz o título do artigo, autor, ano de publicação, periódico de publicação do artigo e, na última coluna por meio de categorias, o(s) fator(es) facilitador(es) do acesso na atenção primária à saúde mencionado(s) nos artigos.

Quadro 1: Quadro síntese dos artigos selecionados para a revisão integrativa segundo título, autor, ano de publicação, periódico e categoria de fatores facilitadores do acesso na atenção primária à saúde.

\begin{tabular}{|c|c|c|c|c|}
\hline Título & Autor & Ano & Periódico & $\begin{array}{l}\text { Fatores } \\
\text { facilitadores do } \\
\text { acesso na atenção } \\
\text { primária à saúde } \\
\text { mencionados no } \\
\text { artigo }\end{array}$ \\
\hline $\begin{array}{lr}\text { Acolhimento } & \text { aos } \\
\text { usuários: uma revisão } & \\
\text { sistemática } & \text { do } \\
\text { atendimento no Sistema } \\
\text { Único de Saúde. }\end{array}$ & $\begin{array}{l}\text { Carvalho et } \\
\text { al, }\end{array}$ & 2008 & $\begin{array}{l}\text { Arquivos de } \\
\text { ciência da Saúde }\end{array}$ & - Acolhimento \\
\hline $\begin{array}{l}\text { Acolhimento na Atenção } \\
\text { Primária à Saúde: revisão } \\
\text { integrativa. }\end{array}$ & $\begin{array}{l}\text { Coutinho et } \\
\text { al. }\end{array}$ & 2015 & Saúde Debate & - Acolhimento \\
\hline $\begin{array}{l}\text { Acesso ao cuidado na } \\
\text { Atenção } \text { Primária à } \\
\text { Saúde } \\
\text { situação, problema e } \\
\text { estratégias de superação. }\end{array}$ & Tesser et al. & 2018 & Saúde Debate & $\begin{array}{l}\text { - Acolhimento } \\
\text { - Estratégia de Saúde } \\
\text { da Família }\end{array}$ \\
\hline
\end{tabular}




\begin{tabular}{|c|c|c|c|c|}
\hline $\begin{array}{l}\text { Avaliação da atenção } \\
\text { básica à saúde sob a ótica } \\
\text { dos usuários: uma } \\
\text { revisão sistemática. }\end{array}$ & Paula et al. & 2016 & $\begin{array}{ll}\text { Revista } & \text { da } \\
\text { escola } & \text { de } \\
\text { enfermagem } & \text { da } \\
\text { USP. } & \end{array}$ & $\begin{array}{l}\text { - Estratégia de Saúde } \\
\text { da Família } \\
\text { - Gerenciamento da } \\
\text { UBS }\end{array}$ \\
\hline $\begin{array}{l}\text { Modos de produzir } \\
\text { cuidado e a } \\
\text { universalidade do acesso } \\
\text { na atenção primária à } \\
\text { saúde }\end{array}$ & $\begin{array}{l}\text { Menezes et } \\
\text { al. }\end{array}$ & 2017 & $\begin{array}{l}\text { Saúde e } \\
\text { Sociedade }\end{array}$ & $\begin{array}{l}\text { - Gerenciamento da } \\
\text { UBS }\end{array}$ \\
\hline $\begin{array}{l}\text { Política de humanização } \\
\text { na atenção básica: } \\
\text { revisão sistemática. }\end{array}$ & Nora et al. & 2013 & $\begin{array}{l}\text { Revista Saúde } \\
\text { Pública }\end{array}$ & $\begin{array}{l}\text { - Gerenciamento da } \\
\text { UBS }\end{array}$ \\
\hline $\begin{array}{l}\text { Acesso aos cuidados } \\
\text { primários de saúde: } \\
\text { revisão integrativa }\end{array}$ & Rocha et al. & 2016 & $\begin{array}{lr}\text { Physis: } & \text { Revista } \\
\text { de } & \text { Saúde } \\
\text { Coletiva } & \end{array}$ & $\begin{array}{l}\text { - Gerenciamento da } \\
\text { UBS }\end{array}$ \\
\hline $\begin{array}{l}\text { Acesso aos serviços da } \\
\text { Atenção Primária em } \\
\text { Saúde: } \\
\text { integrativa da literatura }\end{array}$ & $\begin{array}{l}\text { Figueira et } \\
\text { al. }\end{array}$ & 2018 & $\begin{array}{l}\text { Revista } \\
\text { Brasileira de } \\
\text { Enfermagem }\end{array}$ & $\begin{array}{l}\text { - Gerenciamento da } \\
\text { UBS }\end{array}$ \\
\hline $\begin{array}{l}\text { Desempenho da Atenção } \\
\text { Primária à Saúde } \\
\text { segundo o instrumento } \\
\text { PCA Tool: uma revisão } \\
\text { sistemática }\end{array}$ & Prates et al. & 2017 & $\begin{array}{l}\text { Ciência e Saúde } \\
\text { Coletiva }\end{array}$ & $\begin{array}{l}\text { - Gerenciamento da } \\
\text { UBS }\end{array}$ \\
\hline $\begin{array}{l}\text { Indicadores de qualidade } \\
\text { na atenção primária à } \\
\text { saúde no Brasil: uma } \\
\text { revisão integrativa }\end{array}$ & Ferreira et al. & 2017 & $\begin{array}{l}\text { Revista Ciência } \\
\text { Plural }\end{array}$ & $\begin{array}{l}\text { - Gerenciamento da } \\
\text { UBS }\end{array}$ \\
\hline $\begin{array}{l}\text { A avaliação da atenção } \\
\text { primária a saúde na }\end{array}$ & Silva et al. & 2017 & $\begin{array}{l}\text { Revista } \\
\text { Brasileira de } \\
\text { Enfermagem }\end{array}$ & $\begin{array}{l}\text { - Gerenciamento da } \\
\text { UBS }\end{array}$ \\
\hline
\end{tabular}




\begin{tabular}{|l|l|l|l|l|}
\hline $\begin{array}{l}\text { perspectiva da população } \\
\text { masculina }\end{array}$ & & & & \\
\hline $\begin{array}{l}\text { Avaliação da Atenção } \\
\text { Primária à Saúde sob a } \\
\text { ótica de cuidadores de } \\
\text { crianças: revisão } \\
\text { integrativa da et al. }\end{array}$ & 2019 & Revista de & - Gerenciamento da \\
escola & & Enfermagem da & \\
\hline $\begin{array}{l}\text { Acesso e equidade nos } \\
\text { serviços de saúde: uma } \\
\text { revisão estruturada }\end{array}$ & Barros et al. & 2016 & Saúde Debate & - Redes de Atenção \\
\hline $\begin{array}{l}\text { Revisão sistemática } \\
\text { sobre o conceito de } \\
\text { acesso nos serviços de } \\
\text { saúde: contribuições do } \\
\text { planejamento. }\end{array}$ & & 2010 & Ciência e Saúde & - Redes de Atenção \\
\hline
\end{tabular}

Fonte: Elaboração própria.

Os diversos fatores que facilitam o acesso do usuário a Atenção básica, encontrados nos artigos selecionados, puderam ser classificados em quatro categorias principais como: (i) o acolhimento; (ii) a implantação da Estratégia Saúde da Família (ESF); (iii) a implantação de Redes de Atenção à Saúde; e (iv) o gerenciamento, os quais serão discutidos nas próximas sessões:

\section{Acolhimento nos serviços de saúde pública}

O acolhimento pode ser definido como:

(...) dar acolhida, admitir, aceitar, dar ouvidos, dar crédito a, agasalhar, receber, atender, admitir (BRASIL Apud FERREIRA, 1975). O acolhimento como ato ou efeito de acolher expressa, em suas várias definições, uma ação de aproximação, um "estar com” e um "estar perto de”, ou seja, uma atitude de inclusão. (BRASIL, 2010a, p.6). 
A pesquisa obteve como parte dos resultados, a informação que o acolhimento é um importante fator facilitador do acesso, conforme descrito por Carvalho et al. (2008), Coutinho, Barbieri e Dos Santos (2015) e Tesser, Norman e Vidal (2018).

Nesse sentido, os trabalhos de Carvalho et al. (2008) e Coutinho, Barbieri e Dos Santos (2015) demonstram que o acolhimento é a diretriz com maior evidência dentro da Política Nacional de Humanização (PNH). Segundo este documento, acolher, além de recepcionar o paciente, estabelece uma relação de vínculo e confiança entre o usuário e a equipe.

A PNH deve estar inserida em todos os programas relacionados ao SUS, para promover uma aproximação dos grupos envolvidos, desde o usuário até os profissionais, sejam eles da saúde ou não. Essa comunicação torna-se importante para criação de debates, compartilhamento de experiências e responsabilidades, na perspectiva de melhorar a forma do cuidado e promoção de saúde (BRASIL, 2003).

Além disso, a PNH propõe a construção, de maneira compartilhada, de planos de ação para desenvolver e divulgar inovações no âmbito da saúde. A junção dos três atores - gestores, profissionais e usuários - é fundamental para a humanização na produção de saúde, com a intenção de incluir e estimular novas formas de cuidado (BRASIL, 2013).

Dentro dessa política, o acolhimento é uma das diretrizes que a fortalece, facilitando sua aplicação e a garantia ao acesso à APS. Assim, é possível escutar o usuário, resolver suas queixas mais comuns e referenciá-lo quando necessário, com o intuito de estabelecer uma relação de confiança e fortalecimento de vínculo entre a equipe e o usuário (BRASIL, 2013). É através de uma escuta qualificada, que se permite aos cidadãos o acesso às ações em saúde, ampliando e assegurando de forma efetiva as práticas de saúde (GONÇALVEZ; FIORE, 2012).

Ademais, segundo Tesser, Norman e Vidal (2018) e Carvalho et al. (2008), o acolhimento, através da escuta qualificada, possibilita a ampliação do acesso universal, aumenta a resolutividade, humaniza a relação e agiliza o atendimento.

Neste contexto, o Ministério da Saúde (MS) (2010), desenvolveu uma cartilha sobre o acolhimento nas práticas de produção de saúde. Esta, descreve que o primeiro contato do paciente com o serviço de saúde, favorece a construção da relação de confiança dos usuários com a equipe, corroborando com os resultados do presente estudo (BRASIL, 2010a).

Assim como Coutinho, Barbieri e Dos Santos (2015), o material divulgado pelo MS também aborda, que a organização no atendimento da demanda espontânea e dos pacientes pré- 
agendados, bem como, atividades através de rodas de conversas, grupos de convivência e terapia comunitária, além de ampliarem o acesso, aumentam a satisfação do usuário, com subsequente melhora do cuidado prestado. Tal situação pode ser alcançada, conforme referido por Tesser, Norman e Vidal (2018) e Paula et al. (2016), por meio da Estratégia de Saúde da Família (ESF), que visa promover o atendimento do usuário de forma integral, aproximando a população à equipe da APS.

\section{A Estratégia Saúde da Família no Brasil}

Em 1994, foi desenvolvido o Programa de Saúde da Família (PSF), com o objetivo de aumentar o acesso da população com o serviço de saúde, na atenção primária, o que possibilitou uma mudança no modelo assistencial, como demonstra Pinto e Giovanella (2018). O PSF foi criado para fortalecer a atenção básica, como primeiro nível de atenção à saúde, observando não somente a doença, mas avaliando o ser humano na sua totalidade, além da sua família (DE SOUSA; HAMANN, 2009; DE FARIA et al., 2010), o qual foi se aperfeiçoando e:

(...) a Saúde da Família deixou de ser um programa que operacionalizava uma política de focalização da atenção básica em populações excluídas do consumo de serviços, para ser considerada um estratégia de mudança do modelo de atenção à saúde no SUS, na verdade, o instrumento de uma política de universalização da cobertura da atenção básica e, portanto, um espaço de reorganização do processo de trabalho em saúde nesse nível. Mais que isso, a Saúde da Família vem sendo concebida como parte de uma estratégia maior de mudança do modelo de atenção, na medida que se conjugue com mudanças na organização da atenção de média e alta complexidade induzidas por políticas de regulação e controle. (TEIXEIRA, 2006, p. 63-64)

Em 28 de setembro de 2017 foi sancionada a Portaria de Consolidação No2 que dispõe das normas sobre as políticas nacionais de saúde do Sistema Único de Saúde, fortalecendo e ampliando a Portaria $n^{\circ}$ 648, de 28 de março de 2006, expondo as especificidades da Estratégia Saúde da Família, visando reorganizar a Atenção Básica de acordo com os preceitos do SUS. (BRASIL, 2006; BRASIL, 2017).

Para alcançar esse objetivo é proposto a realização do cadastramento domiciliar no território, diagnóstico situacional, ações de promoção, prevenção e soluções dos problemas de 
saúde, políticas voltadas à saúde de diversos segmentos populacionais, promoção da equidade em saúde, dentre outros, sendo assim, um espaço de construção da cidadania (BRASIL, 2017).

Da mesma forma, a portaria define áreas estratégicas, que devem receber atenção para atuação em todo o território nacional. Tais medidas tem como foco eliminação da hanseníase, controle da tuberculose, hipertensão arterial e diabetes mellitus, além da eliminação da desnutrição infantil, avaliação da saúde da criança, da mulher, do idoso, do negro, abarcando vários seguimentos populacionais, contando com o suporte da promoção da saúde e prevenção de outros agravos, o que pode ser melhor desempenhado pelo nível primário de atenção à saúde com a Estratégia Saúde da Família (BRASIL, 2017).

A Estratégia Saúde da Família foi proposta como modelo de reestruturação dos serviços de saúde e da sua relação com a sociedade, ampliando assim o vínculo com o indivíduo e sua família com consequente melhora da acessibilidade a todos os programas interdisciplinares que são desempenhados pela atenção básica (GUSSO, 2019). Dessa forma, a multidisciplinaridade da equipe amplia o desempenho profissional, integrantes como os Agente Comunitários de Saúde (ACS) por exemplo, passam a ser o elo entre a comunidade do território e o profissionais de saúde, fortalecendo as relações sociais, além do importante papel nas ações de vigilância em saúde, a qual fornece suporte a todos os níveis de saúde, ao apresentar dados epidemiológicos de enfermidades que podem ser identificadas e tratadas clinicamente na atenção básica, não demandando encaminhamento para o nível secundário ou especializado - ambulatorial ou hospitalar (GUSSO, 2019).

Após a implementação do PSF, diversos trabalhos têm sido feitos, a respeito da necessidade de atendimento em serviços de nível secundário, como o trabalho de Paula et al. (2016), que relata um crescimento nas taxas de hospitalizações da população mais vulnerável, que pode ser devido a uma "lacuna no acesso aos serviços preventivos ou o uso de serviços de maior complexidade como substitutos dos cuidados primários". Na mesma linha de pensamento, Alfradique et al. (2009) aponta que a procura pelo atendimento especializado ou pronto-atendimento, sugere falta de acesso à atenção primária, a baixa vinculação ao serviços de atenção primária, a falta de capacidade desse nível de atenção em resolver o problema de saúde ou até mesmo pela atenção tardia que é ofertada. Sendo que a prevenção de doenças, o diagnóstico e o tratamento precoce de patologias agudas diminuiria o risco de internações hospitalares. 
Com isso, a pesquisa de Pinto e Giovanella (2018) demonstra uma redução na taxa de internações, vinculando esse fato ao avanço da cobertura da Estratégia Saúde da Família (ESF), com melhora no acompanhamento dos pacientes com doenças crônicas e a maior facilidade de obtenção de medicamentos e adesão à terapêutica, o que é favorecido pelas redes de atenção em saúde.

\section{Redes de atenção em saúde: a integralidade do cuidado}

Outro fator facilitador do acesso na atenção primária à saúde foi a criação de Redes de Atenção à Saúde (RAS), definidas como "arranjos organizativos de ações e serviços de saúde, de diferentes densidades tecnológicas, que integradas por meio de sistemas de apoio técnico, logístico e de gestão, buscam garantir a integralidade do cuidado" (BRASIL, 2010b, p. 4).

Segundo De Jesus e Assis (2010) e Barros et. al. (2016), a criação dessas redes amplia o acesso e facilitam o uso regular dos serviços de saúde, com equidade, baseado nos princípios fundamentais de regionalização e hierarquização, voltando a perspectiva, continuamente, para o cuidado integral do usuário do serviço de saúde.

As Redes de atenção em saúde têm como objetivo:

(...) promover a integração sistêmica, de ações e serviços de saúde com provisão de atenção contínua, integral, de qualidade, responsável e humanizada, bem como incrementar o desempenho do Sistema, em termos de acesso, equidade, eficácia clínica e sanitária; e eficiência econômica. (BRASIL, 2010b, p. 4)

Dentro das RAS, a Atenção Básica atua como centro de comunicação entre os pontos de atenção, visando contemplar as necessidades de saúde da população através do cuidado multiprofissional e a criação de um sistema logístico e de suporte. Além disso, tem importância na definição da população e território, assim como na avaliação do diagnóstico situacional (BRASIL, 2014). Entretanto, para que as ações de saúde pública sigam o fluxo adequado nas RAS, de forma interdisciplinar, a fim de garantir o cuidado integral, é indispensável que as gestões dos sistemas e dos serviços de saúde, especialmente as das unidades de APS, atuem no planejamento e execução do processo de trabalho eficaz para a garantia dos princípios 
doutrinários do SUS - universalidade, equidade e integralidade - e dos fundamentos da APS primeiro contato/acesso, coordenação do cuidado, integralidade, longitudinalidade, abordagem familiar e enfoque comunitário (FIGUEIRA; DA SILVA; SILVA, 2018).

Todo o processo de cuidado passa por diversos níveis de atenção e complexidade quando necessário. A APS, como coordenadora desse fluxo, facilita o vínculo do usuário com o sistema de saúde através da RAS, promovendo a integralidade do cuidado, já que amplia o acompanhamento daquele usuário, por exemplo, quando o médico da família e comunidade referencia o paciente ao especialista, aguardando a contrarreferência, com a finalidade de propor um acompanhamento longitudinal e evitar que o cliente tenha um fluxo inadequado na rede que vincula todos os níveis de atenção em saúde, fazendo com que o usuário se sinta cada vez mais acolhido e seguro com a equipe. (GUSSO, 2019)

\section{O processo de trabalho que facilita o acesso dos cidadãos ao SUS: Gerenciamento democrático da unidade Básica de Saúde}

O gerenciamento da Unidade Básica de Saúde (UBS) está intimamente relacionado com as RAS, visando coordenar a atenção em saúde, assim como identificar as especificidades do processo de trabalho no âmbito de prestação e promoção de saúde de forma eficaz (DE FARIA et al., 2017).

Para que isso ocorra de maneira propícia à integralidade da atenção ao cidadão, a gestão em saúde deve contemplar os fatores (i) localização geográfica; (ii) dia e horário de funcionamento; (iii) tempo de espera na recepção; (iv) a presença de telefone na unidade; (v) demora na marcação de consultas e (vi) demanda e capacidade no atendimento (MENEZES et al., 2017; PRATES, et al., 2017; FILHO, et al., 2019).

Segundo Menezes et al. (2017), Ferreira et al. (2017), Prates et al. (2017) e Figueira; Da Silva e Silva (2018), a localização da Unidade Básica de Saúde afeta no acesso ao serviço de saúde. Visto que, quando a UBS possui boa localização, proporciona fácil acesso físico e geográfico, facilita a procura da comunidade por atendimento.

Com relação aos dias e horários de funcionamento da Unidade, Prates et al. (2017), Paula et al. (2016) e Silva et al. (2018) apontam a necessidade de ampliar o horário de funcionamento para que a população que trabalha durante o dia, possa buscar atendimento após o expediente, bem como estender os atendimento também ao sábados. 
O longo tempo de espera na recepção para o atendimento médico, foi questionado pelos autores Filho et. al. (2019), Rocha; Bocchi e De Godoy (2016), Prates et al. (2017) e Paula et al. (2016), abordando que a organização com relação a agenda pode diminuir esse transtorno. Além disso, Filho et al. (2019), Paula et al. (2016), Menezes et al. (2017), Prates et al. (2017) e Nora e Jungles (2013) indicam que determinar um número específico de vagas para consultas pré-agendadas, mantendo horários para demanda espontânea, proporciona que o usuário se sinta mais acolhido e com acesso facilitado ao sistema.

Além dos fatores descritos anteriormente, Filho et al. (2019), Ferreira et al. (2017) e Paula et al. (2016) indicam que a presença de um telefone na Unidade de Saúde, proporciona uma melhor aproximação do usuário com a equipe, podendo realizar marcações de consultas e solucionar dúvidas. Dessa forma, facilita o acesso e o vínculo, sem a necessidade de o paciente precisar ir na estrutura física do Posto de Saúde.

Nesse sentido, Rocha; Bocchi e De Godoy (2016), Nora e Jungles (2013) e Menezes et al. (2017) comentam que o rápido fluxo de atendimentos e a resolubilidade promovida pelas atividades em saúde, podem ser alcançadas, por meio da maior disponibilidade de profissionais ou reorganização da demanda da população na procura pelos serviços. $\mathrm{O}$ aumento da demanda, com uma equipe reduzida para realizar os atendimentos, afastam a população, a qual recorre, como porta de entrada, o serviço de urgência e emergência, na tentativa de uma solução rápida ao problema de saúde.

\section{CONCLUSÃO}

As ações de saúde prestadas pelos serviços públicos, tem como objetivo alcançar a população de forma universal, integral e com equidade. Dessa forma, torna-se necessário investir no acesso do paciente aos serviços de saúde, para que este crie vínculo na Unidade Básica de Saúde, fortalecendo a confiança com a equipe, para que assim tenha uma maior adesão ao tratamento sempre que orientado.

A porta de entrada do usuário ao sistema de saúde, inicialmente, deve acontecer através da APS por meio das Redes de Atenção à Saúde, no qual promove a integração entre os diferentes níveis de saúde. Por isso, é tão importante estudar o fluxo e a drenagem de usuários no SUS, identificando fatores facilitadores e dificultadores do acesso às ações fornecidas pela 
política pública de saúde. Dessa forma, os planejamentos de gestão em todos os níveis das Redes de Atenção à Saúde podem ser fundamentados por evidências científicas, reduzindo as desigualdades em saúde.

Nove dos quatorze trabalhos levantados pela pesquisa na literatura científica evidencia que os facilitadores ao acesso do usuário na APS estão relacionados ao gerenciamento da UBS. Assim, os estudos apresentam fatores que podem ser considerados pelos gestores das UBS, facilitando a acessibilidade na APS e nos demais serviços (FILHO et al., 2019; FIGUEIRA; DA SILVA; SILVA, 2018; SILVA et al., 2017; FERREIRA et al., 2017; PRATES et al., 2017; MENEZES et al., 2017; PAULA et al., 2016; ROCHA; BOCCHI; DE GODOY, 2016; NORA; JUNGLES, 2013). Entretanto, ainda faltam pesquisas na área de saúde pública, e delineamentos mais enfáticos para que essas ações contribuam, positivamente o sistema público de saúde. Além disso, é preciso que os profissionais rompam com os paradigmas presentes na prática, que contrapõe a legislação vigente da saúde pública brasileira, tendo mais compromisso com as normativas e delineamento pré-existentes do sistema de saúde.

Nesse sentido, torna-se importante ampliar o atendimento da população, bem como o número de profissionais, em proporção à demanda populacional do território, com capacitação de todos os integrantes das equipes. Assim, pode se alcançar um acolhimento mais empático, justo e equânime à comunidade, além de despender investimentos em práticas de gestão, que fomentem uma maior adesão dos pacientes às ações em saúde promovidas pelo SUS.

\section{REFERÊNCIAS}

AGUIAR, Z. N. SUS: Sistema Único de Saúde - antecedentes, percurso, perspectivas e desafios. 2 ed. São Paulo: Martinari, 2015.

ALFRADIQUE, M. E; BONOLO, P. F; DOURADO, I; LIMA-COSTA, M. F; MACINKO, J; MENDONÇA, C. S; OLIVEIRA, V. B; SAMPAIO, L. F. R; DE SIMONI, C; TURCI, M. A. Internações por condições sensíveis à atenção primária: a construção da lista brasileira como ferramenta para medir o desempenho do sistema de saúde (Projeto ICSAP - Brasil).

Cadernos de Saúde Pública, Rio de Janeiro, v.25, n. 6, p. 1337-1349, 2009.

ASSOCIAÇÃO PAULISTA DE MEDICINA. SUS - O que você precisa saber sobre o Sistema Único de Saúde. São Paulo: Atheneu, 2008. 
BARBOSA, G. C; MENEGUIM, S; LIMA, S. A. M; MORENO, V. Política Nacional de Humanização e formação dos profissionais de saúde: revisão integrativa. Revista Brasileira de Enfermagem, Brasília, v. 66, n. 1, p. 123-127, 2013.

BARROS, F. P. C. D; LOPES, J. D. S; MENDONÇA, A. V. M; SOUSA, M. F. D. Acesso e equidade nos serviços de saúde: uma revisão estruturada. Saúde em Debate, Rio de Janeiro, v. 40, n. 110, p. 264-271, 2016.

BRASIL. [Constituição (1988)]. Constituição da República Federativa do Brasil de 1988. Brasília, DF: Presidência da República, [2016]. Disponível em:

<http://www.planalto.gov.br/ccivil_03/constituicao/constituicao.htm>. Acesso em: 01 out. 2019.

Lei Orgânica da Saúde no 8.080, de 19 de setembro de 1990. Dispõe sobre as condições para a promoção, proteção e recuperação da saúde, a organização e o funcionamento dos serviços correspondentes e dá outras providências. Brasília, DF: Presidência da República, [2017]. Disponível em: < http://www.planalto.gov.br/ccivil_03/leis/18080.htm>. Acesso em: 29 set. 2019.

Ministério da Saúde. Política Nacional de Humanização - Humaniza SUS. 2003. Disponível em: <http://www.saude.gov.br/saude-de-a-z/projeto-lean-nas-emergencias/693acoes-e-programas/40038-humanizasus>. Acesso em: 30 set. 2019.

. Ministério da Saúde. Portaria nº 648, de 28 de março de 2006. Aprova a Política Nacional de Atenção Básica, estabelecendo a revisão de diretrizes e normas para a organização da Atenção Básica para o Programa Saúde da Família (PSF) e o Programa Agentes Comunitários de Saúde (PACS). Diário Oficial da União, 2006. Disponível em: <http://189.28.128.100/dab/docs/legislacao/portaria_648_28_03_2006.pdf >. Acesso em: 05 fev. 2020.

Ministério da Saúde. Acolhimento nas práticas de produção de saúde. $2^{\mathrm{a}}$ ed, $5^{\mathrm{a}}$ reimpressão. Brasília, DF. 2010a. Disponível em:< https://bvsms.saude.gov.br/bvs/publicacoes/acolhimento_praticas_producao_saude.pdf $>$. Acesso em: 19 mar. 2020.

Ministério da Saúde. Portaria no 4.279, de 30 de dezembro de 2010. Estabelece diretrizes para a organização da Rede de Atenção à Saúde no âmbito do Sistema Único de Saúde (SUS). Diário Oficial da União, 2010b. Disponível em: < https://bvsms.saude.gov.br/bvs/saudelegis/gm/2010/prt4279_30_12_2010.html >. Acesso em: 05 fev. 2020.

Ministério da Saúde. Política Nacional de Humanização - PNH. $1^{\text {a }}$ edição. Brasília. 2013. Disponível em: <http://bvsms.saude.gov.br/bvs/publicacoes/politica_nacional_humanizacao_pnh_folheto.pdf >. Acesso em: 30 set. 2019 
Ministério da Saúde. Secretaria de Atenção à Saúde. Implantação das Redes de Atenção à Saúde e outras estratégias da SAS. Brasília: 2014. Disponível em: < https://bvsms.saude.gov.br/bvs/publicacoes/implantacao_redes_atencao_saude_sas.pdf $>$. Acesso em: 29 abr. 2020.

Ministério da Saúde. Portaria de Consolidação nº 2, de 28 de setembro de 2017. Consolidação das normas sobre as políticas nacionais de saúde da Sistema único de Saúde. Diário Oficial da União, 2017. Disponível em: <http://bvsms.saude.gov.br/bvs/saudelegis/gm/2017/prc0002_03_10_2017.html>. Acesso em: 27 ago. 2020.

CARVALHO, C. A. P; MARSICANO, J. Á; CARVALHO, F. S; SALES-PERES, F; BASTOS, J. R. M; SALES-PERES, S. H. C. Acolhimento aos usuários: umas revisão sistemática do atendimento no Sistema único de Saúde. Arquivos de Ciências Saúde, São José do Rio Preto, v. 15, n. 2, p. 93-95, 2008.

CORDOBA, E. SUS e ESF - Sistema único de saúde e estratégia saúde da família. São Paulo. Editora Rideel. 2013.

COUTINHO, L. R. P; BARBIERI, A. R; DOS SANTOS, M. L. M. Acolhimento na Atenção Primária à Saúde: revisão integrativa. Saúde em Debate, Rio de Janeiro, v. 39, n. 105, p. 514524, 2015.

DE FARIA, H. P; COELHO, I. B; WERNECK, M. A. F.; SANTOS, M. A. Modelo assistencial e atenção básica em saúde. $2^{\mathrm{a}}$ ed. Belo Horizonte: NESCON/UFMG/COOPEMED. 2010. Disponível em: <https://www.nescon.medicina.ufmg.br/biblioteca/imagem/1792.pdf >. Acesso em: 07 fev. 2020.

DE FARIA, H. P; WERNECK, M. A. F; DOS SANTOS, M.A; TEIXEIRA, P.F. Processo de trabalho em saúde. $3^{\text {a }}$ ed. Belo Horizonte: Nescon/UFMG. 2017. Disponível em:

<https://www.nescon.medicina.ufmg.br/biblioteca/imagem/Processo-trabalho-Saude-ModuloAtencao.pdf $>$. Acesso em: 18 abr. 2020

DE JESUS, W. L. A; ASSIS, M. M. A. Revisão sistemática sobre o conceito de acesso nos serviços de saúde: contribuições do planejamento. Ciência \& Saúde Coletiva, Rio de Janeiro, v. 15, n. 1 , p. 161-170, 2010.

DE MATTOS, R. A. Princípios do Sistema Único de Saúde (SUS) e a humanização das prática de saúde. Interface - Comunicação, saúde, educação, Botucatu, v. 13, p. 771-780, 2009.

DE SOUSA, Maria Fátima; HAMANN, Edgar Merchán. Programa Saúde da Família no Brasil: uma agenda incompleta? Ciência \& Saúde Coletiva [on line], Rio de Janeiro, v. 14, p. 1325-1335, 2009. 
DE SOUZA, R. R. O sistema público de saúde brasileiro. Brasília, DF: Ministério da Saúde. 2002.

FERREIRA, J. M; KULBOK, P; DA SILVA, C. A. B; DE ANDRADE, F. B; COSTA, I. C. C. Indicadores de qualidade na Atenção Primária à Saúde no Brasil: uma revisão integrativa. Ciência Plural, Rio Grande do Norte, v. 3, n. 3, p. 45-68, 2017.

FIGUEIRA, M. C. S; DA SILVA, W. P; SILVA, E. M. Acesso aos serviços da Atenção Primária em Saúde: revisão integrativa da literatura. Revista Brasileira de Enfermagem, Brasília, v. 71, n. 3, p. 1245-1257, 2018.

FILHO, A. C. A. A; SILVA, N. A; RIBEIRO, M. G. C; DA ROCHA, S. S; ANDRADE, E. M. L. R; NOGUEIRA, L.T. Avaliação da Atenção Primária à Saúde sob a ótica de cuidadores de crianças: revisão integrativa. Revista de Enfermagem da USP, São Paulo, v. 53, p. 1-9, 2019.

GONÇALVEZ, D. A; FIORE, M. L. M. Vínculo, acolhimento e abordagem psicossocial: a prática da integralidade. Curso de Especialização em Saúde da Família - UMASUS/UNIFESP. 2012.

GUSSO, G; LOPES, J. M. C. Tratado de medicina de família e comunidade: princípios, formação e prática. 2 ed. Porto Alegre: Artmed, 2019.

LIMA, S. A. V; DA SILVA, M. R. F; DE CARVALHO, E. M. F; CESSE, E. Â. P; DE BRITO, E. S. V; BRAGA, J. P. R. Elementos que influenciam o acesso à atenção primária na perspectiva dos profissionais e dos usuários de uma rede de serviços de saúde do Recife. Physis: Revista de Saúde Coletiva, Rio de Janeiro, v. 25, n. 2, p. 635-656, 2015.

MENDES, I. A. C. Desenvolvimento e saúde: a declaração de Alma-Ata e movimentos posteriores. Revista Latino-Americana de enfermagem, Ribeirão Preto, v. 12, n. 3, p. 447448, 2004.

MENDES, E.V. As redes de atenção à saúde. Ciência \& Saúde Coletiva, Rio de Janeiro, v. 15, n. 5, p. 2297-2305, 2010.

MENEZES, E. L. C; SCHERER, M. D. A; VERDI, M. I; PIRES, D. P. Modos de produzir cuidado e a universalidade do acesso na atenção primária à saúde. Saúde $\mathbf{e}$ Sociedade, São Paulo, v. 26, n. 4, p. 888 - 899, 2017.

NORA, C. R. D; JUNGES, J. R. Política de humanização na atenção básica: revisão sistemático. Revista de Saúde Pública [online], São Paulo, v. 47, n. 6, p. 1186-1200, 2013.

PAULA, W. K. A. S. D; SAMICO, I. C; CAMINHA, M. D. F. C; DA SILVA, S. L. Avaliação da atenção básica à saúde sob a ótica dos usuários: uma revisão 
sistemática. Revista da Escola de Enfermagem da USP, São Paulo, v. 50, n. 2, p. 335-345, 2016.

PINTO, L. F; GIOVANELLA, L. Do Programa à Estratégia Saúde da Família: expansão do acesso e redução das internações por condições sensíveis à atenção básica (ICSAB). Ciênc. saúde coletiva [online], Rio de Janeiro, v. 23, n. 6, p. 1903-1914, 2018.

PRATES, M. L; MACHADO, J. C; DA SILVA, L.S; AVELAR, P. S; PRATE, L. L; DE MENDONÇA, E.T; DA COSTA, G. D; COTTA, R. M. M. Desempenho da Atenção Primária à Saúde segundo o instrumento PCATool: uma revisão sistemática. Ciência e Saúde Coletiva, Rio de Janeiro, v. 22, n. 6, p. 1881-1892, 2017.

ROCHA, S. A; BOCCHI, S. C. M; DE GODOY, M. F. Acesso aos cuidados primários de saúde: revisão integrativa. Physis: Revista de Saúde Coletiva, Rio de Janeiro, v. 26, n. 1, p. 87-111, 2016.

SILVA, G. M; RASERA, E. F. A construção do SUS-problema no jornal Folha de S.Paulo. História, ciência, saúde-Manguinhos, Rio de Janeiro, v. 21, n. 1, p. 61-76, 2014.

SILVA, A. N; DA SILVA, S. A; DA SILVA, A. R. V; DE ARAUJO, T. M. E; REBOUÇAS, C. B. A; NOGUEIRA, L. T. A avaliação da atenção primária à saúde na perspectiva da população masculina. Revista Brasileira de Enfermagem, Brasília, v. 71, n. 2, p. 236-243, 2018.

STARFIELD, Bárbara. Atenção primária: equilíbrio entre necessidades de saúde, serviços e tecnologia. Brasília. UNESCO, Ministério da Saúde, 2002.

TEIXEIRA, C.F; SOLLA, J.P. Modelo de atenção à saúde: vigilância e saúde da família [online]. Salvador: Editora EDUFBA, 2006. 237 p. Saladeaula series, n³. ISBN 85-2320400-8. Disponível em: 〈http://books.scielo.org/id/f7/pdf/teixeira-9788523209209-04.pdf〉. Acesso em: 09 mar. 2020.

TESSER, C. D; NORMAN, A. H; VIDAL, T. B. Acesso ao cuidado na Atenção Primária à Saúde brasileira: situação, problemas, e estratégias de superação. Saúde em Debate, Rio de Janeiro, v. 42, n. 1, p. 361-378, 2018.

\section{Declaração de Interesse}

Os autores declaram não haver nenhum

conflito de interesse

Financiamento

Financiamento próprio

\section{Colaboração entre autores}

N.M.R. pesquisou e fez olevantamento bibliográfico, redação, elaboração $e$ submissão do artigo. M. F. O. e L. S. e S. contribuíram no conteúdo intelectual do manuscrito, no desenho e interpretação dos dados e revisão da escrita do manuscrito. 